\title{
Modulating process parameters to change physical properties of bigels for food applications
}

\author{
L.H. Fasolin ${ }^{\mathrm{a}, *}$, A.J. Martins ${ }^{\mathrm{a}, \mathrm{b}}$, M.A. Cerqueira ${ }^{\mathrm{b}}$, A.A. Vicente ${ }^{\mathrm{a}}$ \\ ${ }^{a}$ Centre of Biological Engineering, University of Minho, Campus de Gualtar, 4710-057 Braga, Portugal \\ ${ }^{\mathrm{b}}$ International Iberian Nanotechnology Laboratory, Av. Mestre José Veiga s/n, 4715-330 Braga, Portugal
}

\section{A R T I C L E I N F O}

\section{Keywords:}

Oleogel

Hydrogel

Hybrid gel

Mechanical properties

Rheology

\begin{abstract}
A B S T R A C T
The effect of the process parameters involved in the production of bigels was investigated through the study of structural, mechanical and rheological properties. Four level $\left(2^{4}\right)$ Central Composite Rotational Design (CCRD) configuration was applied in order to study the organogelator concentration, hydrocolloid concentration, organogel:hydrogel ratio and shear of mixing. Gellan gum and high oleic sunflower oil with glycerol monostearate were used to produce hydrogels and organogels, respectively. All formulations were water-in-oil systems with gel-like behavior. FTIR and XRD results showed that bigels were formed only by physical arrangement with no chemical interactions, but gellan gum decreased the crystallinity of the systems due to its amorphous character. However, hydrogel particles acted as an active filler, reinforcing the structure in comparison to pure gels. Also, the glycerol monostearate present in the organogel could self-assemble at the interface in order to interact with the aqueous phase, improving the interaction and affinity between the phases. Moreover, depending on the composition, systems could be softer or harder and present some frequency dependence. CCRD showed that all parameters evaluated changed with organogel:hydrogel ratio and organogel concentration. Shear of mixing was also significant for some parameters; however, hydrocolloid concentration did not exert a significant effect on the range studied. From PCA analysis it was possible to distinguish different groups, which means that some conditions produced bigels with similar characteristics. Thus, it is expected that these results will allow customizing and fine-tuning properties of structured two-phase systems for diverse applications, ranging from food to pharmaceutical and cosmetic industries.
\end{abstract}

\section{Introduction}

Bigels or hybrid gels are novel complex biphasic systems composed by two different semi-solid networks and was first mentioned by Almeida et al. (2008) that mixed an organogel with a hydrogel, naming the result as "bigel". Since then, the interest in such systems has increased due to their physicochemical and functional properties (Deng et al., 2015; Kodela et al., 2017; Patel, Mankoč, Bin Sintang, Lesaffer, \& Dewettinck, 2015; Wakhet et al., 2015). The main advantage of bigels is their ability to simultaneously act as a vehicle for hydrophilic and hydrophobic compounds (Behera, Sagiri et al., 2015; Rehman \& Zulfakar, 2014). In addition, the semi-solid nature of both phases confers to these gels higher thermodynamic and kinetic stability in comparison to other biphasic systems such as emulsions and emulgels (Kodela et al., 2017; Satapathy et al., 2015; Wakhet et al., 2015). These characteristics, allied to the possibility of tailoring the physical properties of the final gel by modifying the single phases properties and their relative ratio made hybrid gelled systems being of great interest for pharmaceutics, cosmetic and food industries.

Despite recent studies on the use of these gels, most publications are focused on pharmaceutical and cosmetic applications, meaning that research is still needed towards their application in foods. In this case, the Generally Recognized as Safe (GRAS) status of the ingredients used to produce bigels is of particular relevance. Different combinations of hydrocolloids, organogelators and oil were studied. Guar gum (Sahoo et al., 2015; Vinay K. Singh, Banerjee et al., 2014), gelatin (Sai Sateesh Sagiri, Singh, Kulanthaivel et al., 2015; Satapathy et al., 2015), gelatin-agar mixture (Wakhet et al., 2015), sodium alginate (Rehman, Mohd Amin, \& Zulfakar, 2014) starch (Behera, Sagiri, Singh, Pal, \& Anis, 2014) and synthetic polymers (PVA and PVP) (Behera, Singh et al., 2015) are examples of hydrocolloids used. In the same way, sorbitan monopalmitate and sunflower oil (Behera, Dey, Sharma, \& Pal, 2015),

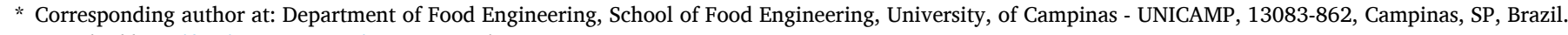

E-mail address: 1fasolin@unicamp.br (L.H. Fasolin).
} 
sorbitan monostearate and sesame oil (Vinay K. Singh, Anis et al., 2014; Vinay K. Singh, Banerjee et al., 2014), policosanol from rice bran wax and glyceril stearate with extra virgin olive oil (Lupi et al., 2016) and medium chain triglycerides with beeswax (Martins et al., 2019) were some of the studied organogels.

However, independently of the system used, all the cited works discuss the mechanical, rheological, and microscopic characteristics of bigels and/or their influence in their release properties. A recent review by Shakeel et al. (2019) highlights the importance of process parameters and other variables, such as storage. However, with the exception of some works that evaluate the hydrogel:organogel ratio and/or the concentration of the gelators (Kodela et al., 2017; Lupi et al., 2015; Lupi, De Santo, Ciuchi, Baldino, \& Gabriele, 2016, 2017; Patel et al., 2015; Rehman \& Zulfakar, 2017; Rehman et al., 2014; Singh, Anis et al., 2014; Singh, Banerjee et al., 2014), no publications could be found considering all the process parameters simultaneously. For example, Lupi et al. (2017) proposed an empirical model to describe rheological properties as a function of single components, evaluating different hydrogel/organogel ratio and different hydro and organogelator concentrations. However, they did not include mixing parameters as a variable. The gels' ratio, the strength of both gels and the energy density applied (i.e. shear) during bigels production can influence the mixing and consequently the final properties of the systems (Shakeel et al., 2019). If such properties are to be tailored according to the foreseen application, it is essential to know the process and to identify the relevant parameters and their influence in the process and product. This lack of information and knowledge about these aspects, reflects the initial stage of this concept and the need for extensive exploitation and characterization of the bigels in order to improve their use as matrices for food, cosmetic and pharmaceuticals applications.

The goal of this work was evaluating the effect of the process variables - hydrocolloid and organogelator concentration; hydrogel:organogel ratio and energy density (evaluated as mixing rate) - on the physical properties (structural, rheological and mechanical) of bigels. This will allow fine tuning these properties in order to use these systems for different applications, especially in foods. For this purpose, hydrogels were produced with gellan gum (GG), while for organogels high oleic sunflower oil (HOSO) and glycerol monostearate (GM) was used. Bigels were evaluated through rheological, mechanical and microscopic analysis.

\section{Material and methods}

\subsection{Material}

Deacylated gellan gum (GG) powder (Kelcogel ${ }^{\circledR}$ F) was kindly donated by CP Kelco (Brazil) and high oleic sunflower oil (HOSO - 80.1 $\%$ of $\mathrm{C} 18: 1,10.7 \%$ of C18:2) by Cargill (Belgium). Glycerol monostearate (GM) (>95.0\%) was purchased from Alfa Aesar (USA)

\subsection{Central composite rotatable design CCRD}

A Central Composite Rotatable Design CCRD $\left(2^{4}\right.$ factorial design with 8 trials under the axial conditions and 3 repetitions at the central point) with 27 trials was performed to evaluate the effects of process conditions (independent variables - organogelator concentration, hydrocolloid concentration, organogel:hydrogel ratio and mixing rate) on physical properties (dependent variables - particle size distribution, rheological and mechanical properties) of the bigels. The range and the levels of the independent variables are shown in Table 1 and the complete CCRD matrix with the responses is presented in Table 3. From the results, second-order models were obtained and evaluated statistically by analysis of variance (ANOVA) using the software Statistica 10 (Statsoft, USA). The selected results were further analyzed by principal component Analysis (PCA) (Section 2.7).
Table 1

Coded and uncoded values of the independent variables used to prepare the bigels.

\begin{tabular}{|c|c|c|c|c|c|}
\hline \multirow{2}{*}{ Independent variables } & \multicolumn{5}{|l|}{ Levels } \\
\hline & -2 & -1 & 0 & +1 & +2 \\
\hline GM concentration $(\% \mathrm{w} / \mathrm{w})$ & 5 & 7.5 & 10 & 12.5 & 15 \\
\hline GG concentration $(\% \mathrm{w} / \mathrm{w})$ & 1.0 & 1.125 & 1.25 & 1.375 & 1.5 \\
\hline Organogel:Hydrogel & $20: 80$ & $35: 65$ & $50: 50$ & $65: 35$ & $80: 20$ \\
\hline Mixing rate $\left(\mathrm{min}^{-1}\right)$ & 500 & 750 & 1000 & 1250 & 1500 \\
\hline
\end{tabular}

*GG - Deacylated gellan gum; GM - Glycerol monostearate.

\subsection{Bigel production}

Hydrogels and organogels were prepared separately. Aqueous GG and GM + HOSO solutions were prepared according to concentrations described in Tables 1 and 2. The solutions were heated at $80{ }^{\circ} \mathrm{C}$ during 30 min under magnetic stirring and cooled to room temperature $\left(25^{\circ} \mathrm{C}\right.$ $\pm 2{ }^{\circ} \mathrm{C}$ ) during $2 \mathrm{~h}$ to ensure gel formation. The verification of selfsustaining systems formation was performed by simple tube flipping.

After the organogel and hydrogel preparation, all the bigels (batches of $100 \mathrm{~g}$ ) were prepared by slowly incorporating the amount of hydrogel into the organogel at room temperature, under mechanical stirring at a pre-determined mixing rate (Tables 1 and 2) using an overhead stirrer Hei-Torque 400 and PR 30 Pitched-Blade Impeller (Heidolph, Germany). Time of mixing was set to $10 \mathrm{~min}$ in order to eliminate this variable. So, with constant volume and time, energy density was dependent only on mixing rate. Systems were stored at room temperature during $24 \mathrm{~h}$ before being analyzed. The physical properties of bigels were compared with pure organogel and hydrogel. However, pure gels were evaluated according to their original (i.e. after gel preparation) and mixed structure to simulate the effect of bigels preparation. For this purpose, $100 \mathrm{~g}$ of organogel or hydrogel was mixed at $1000 \mathrm{~min}^{-1}$ (central point) during $10 \mathrm{~min}$ and defined as Gmix.

\subsection{Fourier transform infrared (FTIR)}

FTIR spectra were recorded with a FT-IR VERTEX 80/80v (Bruker, USA) in Attenuated Total Reflectance mode (ATR) with a platinum crystal accessory in the wavenumber range: $4000-400 \mathrm{~cm}^{-1}$, using 32 scans at a resolution of $4 \mathrm{~cm}^{-1}$. Before analysis, an open bean background spectrum was recorded as a blank. The tests were performed at room temperature $\left(25^{\circ} \mathrm{C} \pm 2{ }^{\circ} \mathrm{C}\right.$ ) (Table 4).

\subsection{X-ray diffraction $(X R D)$}

The X-ray diffraction data were acquired with an X-Ray Diffractometer X'Pert PRO MRD (PanAnalytical, The Netherlands). Scans ranging from 5.0 to $50^{\circ}(2 \theta)$ were performed at room temperature (ca. $\left.22^{\circ} \mathrm{C}\right)$ using an X-ray tube Cu source $(\lambda=1.54056 \AA)$ at $45 \mathrm{kV}$ and $40 \mathrm{~mA}$ with calibration offset for $2 \theta=-0.0372^{\circ}$. Information was collected

Table 2

Rheological and mechanical parameters for pure hydrogel (GG) and organogel (GM) before and after shearing (GMmix) at $1000 \mathrm{~min}^{-1}$ for $10 \mathrm{~min}$. Adhesiveness $(A d)$, hardness $(H d)$, work of shear $(W s)$, $\tan \delta(\tan )$ at $1 \mathrm{~Hz}$, complex modulus $\left(G^{*}\right)$ at $1 \mathrm{~Hz}$.

\begin{tabular}{llllll}
\hline Formulation & Ad (N.s) & Hd (N) & Ws (N.s) & $\tan (-)$ & $\mathrm{G}^{*}(\mathrm{~Pa})$ \\
\hline $1 \% \mathrm{GG}$ & -0.31 & 0.55 & 0.54 & 0.69 & 14.3 \\
$1.5 \% \mathrm{GG}$ & -0.78 & 1.21 & 1.26 & 0.24 & 199 \\
$5 \% \mathrm{GM}$ & -0.57 & 0.85 & 0.90 & 0.26 & 1913 \\
$10 \% \mathrm{GM}$ & -3.89 & 4.67 & 5.87 & 0.26 & 16964 \\
$15 \% \mathrm{GM}$ & -5.13 & 9.35 & 13.11 & 0.27 & 49647 \\
$5 \% \mathrm{GMmix}$ & -0.14 & 0.18 & 0.17 & 0.48 & 202 \\
$10 \% \mathrm{GMmix}$ & -0.19 & 0.34 & 0.36 & 0.44 & 337 \\
$15 \% \mathrm{GMmix}$ & -0.70 & 1.27 & 1.43 & 0.38 & 1571 \\
\hline
\end{tabular}


Table 3

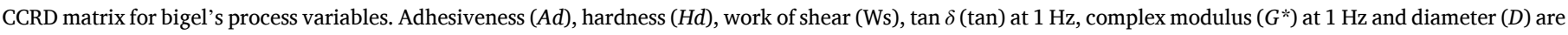

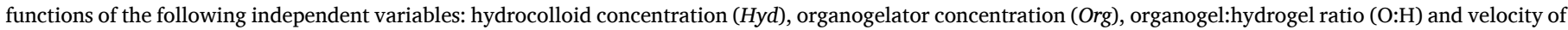
mixing (vel). Independent variables are presented with their coded and real (in brackets) values.

\begin{tabular}{|c|c|c|c|c|c|c|c|c|c|c|}
\hline Trial & Hyd (\%) & Org (\%) & $\mathrm{O}: \mathrm{H}$ & Vel $\left(\min ^{-1}\right)$ & Ad (N.s) & Hd $(\mathrm{N})$ & Ws (N.s) & $\tan (-)$ & $\mathrm{G}^{*}(\mathrm{~Pa})$ & $\mathrm{D}(\mu \mathrm{m})$ \\
\hline 1 & $-1(1.125)$ & $-1(7.5)$ & $-1(35: 65)$ & $-1(750)$ & -1.20 & 2.07 & 1.79 & 0.38 & 2254 & 17.9 \\
\hline 2 & $1(1.375)$ & $-1(7.5)$ & $-1(35: 65)$ & $-1(750)$ & -2.02 & 3.14 & 3.76 & 0.31 & 5199 & 18.8 \\
\hline 3 & $-1(1.125)$ & $1(12.5)$ & $-1(35: 65)$ & $-1(750)$ & -2.28 & 2.97 & 3.05 & 0.41 & 2257 & 16.0 \\
\hline 4 & $1(1.375)$ & $1(12.5)$ & $-1(35: 65)$ & $-1(750)$ & -1.64 & 2.34 & 2.57 & 0.40 & 2411 & 18.1 \\
\hline 5 & $-1(1.125)$ & $-1(7.5)$ & $1(65: 35)$ & $-1(750)$ & -2.28 & 2.97 & 3.05 & 0.26 & 14538 & 16.7 \\
\hline 6 & $1(1.375)$ & $-1(7.5)$ & $1(65: 35)$ & $-1(750)$ & -2.87 & 3.74 & 4.39 & 0.27 & 15874 & 19.1 \\
\hline 7 & $-1(1.125)$ & $1(12.5)$ & $1(65: 35)$ & $-1(750)$ & -5.36 & 7.87 & 9.52 & 0.29 & 39181 & 14.5 \\
\hline 8 & $1(1.375)$ & $1(12.5)$ & $1(65: 35)$ & $-1(750)$ & -6.92 & 10.75 & 12.18 & 0.31 & 34005 & 15.6 \\
\hline 9 & $-1(1.125)$ & $-1(7.5)$ & $-1(35: 65)$ & $1(1250)$ & -1.09 & 1.68 & 1.72 & 0.30 & 10531 & 18.5 \\
\hline 10 & $1(1.375)$ & $-1(7.5)$ & $-1(35: 65)$ & $1(1250)$ & -1.25 & 2.18 & 2.05 & 0.37 & 1631 & 15.9 \\
\hline 11 & $-1(1.125)$ & $1(12.5)$ & $-1(35: 65)$ & $1(1250)$ & -1.82 & 3.05 & 2.73 & 0.47 & 3432 & 24.8 \\
\hline 12 & $1(1.375)$ & $1(12.5)$ & $-1(35: 65)$ & $1(1250)$ & -2.00 & 3.14 & 2.86 & 0.46 & 2728 & 24.6 \\
\hline 13 & $-1(1.125)$ & $-1(7.5)$ & $1(65: 35)$ & $1(1250)$ & -3.14 & 4.14 & 4.95 & 0.27 & 20969 & 15.3 \\
\hline 14 & $1(1.375)$ & $-1(7.5)$ & $1(65: 35)$ & $1(1250)$ & -3.75 & 5.05 & 6.00 & 0.26 & 21991 & 18.5 \\
\hline 15 & $-1(1.125)$ & $1(12.5)$ & $1(65: 35)$ & $1(1250)$ & -6.93 & 12.81 & 15.18 & 0.30 & 62111 & 15.7 \\
\hline 16 & $1(1.375)$ & $1(12.5)$ & $1(65: 35)$ & $1(1250)$ & -8.06 & 12.69 & 14.60 & 0.30 & 64176 & 9.1 \\
\hline 17 & $-2(1.0)$ & $0(10.0)$ & $0(50: 50)$ & $0(1000)$ & -6.00 & 8.40 & 9.93 & 0.40 & 26842 & 15.3 \\
\hline 18 & $2(1.5)$ & $0(10.0)$ & $0(50: 50)$ & $0(1000)$ & -5.50 & 8.50 & 9.92 & 0.46 & 27744 & 15.6 \\
\hline 19 & 0 (1.25) & $-2(5.0)$ & $0(50: 50)$ & $0(1000)$ & -2.13 & 3.93 & 3.55 & 0.20 & 11078 & 24.8 \\
\hline 20 & $0(1.25)$ & $2(15.0)$ & $0(50: 50)$ & $0(1000)$ & -6.91 & 9.74 & 12.09 & 0.48 & 27270 & 16.0 \\
\hline 21 & $0(1.25)$ & $0(10.0)$ & $-2(20: 80)$ & $0(1000)$ & -1.07 & 1.75 & 1.52 & 0.49 & 315 & 27.0 \\
\hline 22 & $0(1.25)$ & $0(10.0)$ & $2(80: 20)$ & $0(1000)$ & -7.12 & 11.84 & 12.79 & 0.24 & 58315 & 18.0 \\
\hline 23 & $0(1.25)$ & $0(10.0)$ & $0(50: 50)$ & $-2(500)$ & -3.87 & 5.89 & 6.14 & 0.31 & 17953 & 16.2 \\
\hline 24 & 0 (1.25) & $0(10.0)$ & $0(50: 50)$ & $2(1500)$ & -4.53 & 6.90 & 7.15 & 0.31 & 28863 & 16.5 \\
\hline 25 & $0(1.25)$ & $0(10.0)$ & $0(50: 50)$ & $0(1000)$ & -6.32 & 9.08 & 10.65 & 0.40 & 13799 & 15.9 \\
\hline 26 & $0(1.25)$ & $0(10.0)$ & $0(50: 50)$ & $0(1000)$ & -6.00 & 9.51 & 11.03 & 0.38 & 14126 & 16.8 \\
\hline 27 & 0 (1.25) & $0(10.0)$ & $0(50: 50)$ & $0(1000)$ & -6.61 & 8.50 & 10.22 & 0.25 & 12618 & 16.2 \\
\hline
\end{tabular}

Table 4

Empirical coded models (statistically significant parameters) and the related ANOVA for the bigel's process variables.

\begin{tabular}{|c|c|c|c|c|c|c|}
\hline \multicolumn{2}{|l|}{ Response } & \multirow{2}{*}{$\begin{array}{l}\text { Eq. } \\
(2)\end{array}$} & \multirow{2}{*}{$\begin{array}{l}\mathrm{R}^{2} \\
0.915\end{array}$} & \multirow{2}{*}{$\begin{array}{l}\mathrm{F}_{\text {calc }} \\
24.12\end{array}$} & \multirow{2}{*}{$\frac{\mathrm{F}_{\mathrm{tab}}}{2.6}$} & \multirow{2}{*}{$\begin{array}{l}\left(\mathrm{DF}_{\mathrm{Reg}}, \mathrm{DF}_{\mathrm{Res}}\right) \\
6,20\end{array}$} \\
\hline Adhesiveness & $A d=-6.31-1.12[\mathrm{Org}]+0.67[\mathrm{Org}]^{2}-1.59(\mathrm{O}: H)+0.78(\mathrm{O}: H)^{2}+0.75 \mathrm{mix}^{2}-0.82[\mathrm{Org}](\mathrm{O}: H)$ & & & & & \\
\hline Hardness & $H d=9.03+1.76[\mathrm{Org}]-0.90[\mathrm{Org}]^{2}+2.49(\mathrm{O}: H)-0.91(\mathrm{O}: H)^{2}-1.0 \mathrm{mix}^{2}+1.61[\mathrm{Org}](\mathrm{O}: H)$ & $(3)$ & 0.916 & 21.27 & 2.6 & 6,20 \\
\hline Work of shear & $W s=10.62+2.17[\mathrm{Org}]-1.07[\mathrm{Org}]^{2}+3.0(\mathrm{O}: H)-1.24(\mathrm{O}: H)^{2}-1.37 \mathrm{mix}^{2}+1.95[\mathrm{Org}](\mathrm{O}: H)$ & (4) & 0.937 & 26.26 & 2.6 & 6,20 \\
\hline $\tan \delta$ & $\tan \delta=0.34+0.047[\mathrm{Org}]^{2}-0.056(\mathrm{O}: H)$ & (5) & 0.822 & 30.91 & 3.4 & 2,24 \\
\hline $\begin{array}{l}\text { Complex } \\
\text { modulus }\end{array}$ & $G^{*}=13514+6237[\operatorname{Org}]+14933(O: H)+2976(O: H)^{2}+3903 \operatorname{mix}+8431[\operatorname{Org}](O: H)+3716 \operatorname{mix}(O: H)$ & $(6)$ & 0.951 & 38.47 & 2.6 & 6,20 \\
\hline Diameter $\left(d_{3,2}\right)$ & $D=16.33-2.0(O: H)+1.35(O: H)^{2}-1.69[\operatorname{Org}](O: H)$ & (7) & 0.781 & 8.04 & 3.03 & 3,23 \\
\hline
\end{tabular}

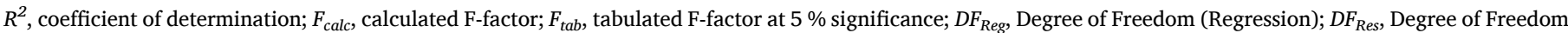
(Residues).

during 174 s. PANalytical X'Pert HighScore Plus Software was used to analyze all XRD data. Determination of the lattice parameter $d$ was performed using Bragg's law.

\subsection{Rheological analyses}

Rheological measurements were performed in a HR-1 (TA Instruments, USA) rheometer equipped with a stainless-steel cone-plate geometry $\left(60 \mathrm{~mm}, 2^{\circ}\right.$ angle, truncation $\left.64 \mu \mathrm{m}\right)$ in triplicate at $25{ }^{\circ} \mathrm{C} \pm$ $0.5{ }^{\circ} \mathrm{C}$. The samples were handled gently to avoid structural damage. Viscoelastic properties were evaluated by oscillatory measurements, using a frequency sweep between 0.1 and $10 \mathrm{~Hz}$ within the linear viscoelasticity region $(0.1 \%$ deformation). The linear viscoelasticity region was determined by strain sweep between $0.01 \%$ and $5 \%$ strain (data not shown). Complex moduli $(G *)$ moduli and $\tan \delta$ were evaluated.

\subsection{Mechanical properties}

Mechanical properties were determined by uniaxial compression measurements using a TA.HDPlus Texture Analyzer (Stable Micro
Systems, UK) with an HDP/SR spreadability rig with $45^{\circ}$ conical Perspex probe. The probe penetrated $23 \mathrm{~mm}$ into the samples using a crosshead speed of $1 \mathrm{~mm} / \mathrm{s}$ and a post-test speed of $10 \mathrm{~mm} / \mathrm{s}$. Mechanical parameters (hardness - maximum force peak, work of shear - positive area and adhesiveness - negative area) were analyzed from the resultant force - time plots. Four replicates were made per sample

\subsection{Optical microscopy}

The bigels were observed under an epifluorescence microscope (Olympus BX51) coupled with a DP72 digital camera and three sets of filters (DAPI - 360-370/420; FITC - 470-490/520; and TRITC - 530550/590) (Olympus Portugal SA, Porto, Portugal) to evaluate the particle size distribution and the organization of the oil and water phases. In order to confirm the structure organization, the conductivity of bigels was measured with a Hanna edge conductivity meter with a HI763100 probe (Hanna Instruments, Italy).

The size of the particles was analyzed using the Olympus cellSens software. At least 500 particles were used to calculate the mean diameter and particle size distribution. The volume-surface mean diameters $\left(d_{32}\right)$ was calculated using Eq. 1. 
$d_{32}=\frac{\sum_{i} n_{i} d_{i}^{3}}{\sum_{i} n_{i} d_{i}^{2}}$

where $n_{i}$ was the number of particles with diameter $d_{i}$.

\subsection{Principal component analysis (PCA)}

The systems produced through CCRD combinations were subjected to principal component analysis (PCA) using the software Statistica 10 (Statsoft, USA).

\section{Results and discussion}

\subsection{Properties of pure organogels and hydrogels}

Organogels or oleogels are semi-solid systems resulting from the immobilization of an organic liquid in a three-dimensional crystalline network formed by an organogelator (Toro-Vazquez et al., 2013). A low intensity broad peak was identified for GM. GMmix and bigels' (almost inexistent) spectra (Fig. 1A). This amorphous peak, around $19.8^{\circ} 2 \theta$, is a result of the presence of triacylglycerols (amorphous scattering of the oil), entrapped within oleogel structure (Sagiri, Singh, Pal, Banerjee, \& Basak, 2015; Trujillo-Ramírez, Lobato-Calleros, Jaime Vernon-Carter, \& Alvarez-Ramirez, 2019). Previous works reported on the spectra of GM, where sharp peaks at $19.6^{\circ}$ and $23.7^{\circ} 2 \theta$ were observed, hence indicating a reorganization when oleogels are formed, resulting in a different molecular packing (Carrillo-Navas, Pérez-Alonso, Fouconnier, Vernon-Carter, \& Alvarez-Ramírez, 2014; Dassanayake, Kodali, Ueno, \& Sato, 2009). When triacylglycerols crystallize, they pack in specific structural arrangements (polymorphism); d-spacings of 3.9-4.4 $\AA$, observed for GM and comparably for GMmix, point to the characteristic $\beta$-phase crystals, with orthorhombic packing of fatty alcohol molecules (Dassanayake et al., 2009; Trujillo-Ramírez et al., 2019). Very slight diffraction intensities were observed for bigel samples with increasing GM content, that would dissipate with cumulative aqueous content within the mixture. The impacts of a decreased solids (GM) content together with increasing aqueous (amorphous) phase and shearing process, seem to have influenced the crystalline content, influencing the overall spectra intensity.

FTIR analyzes (Fig. 1B) were also performed for the pure gels in order to evaluate the interactions between hydrogel and organogels in the hybrid systems. In FTIR spectrum of the pure organogel absorption peaks related to oil and organogelator were very similar, since GM is also derived from triglycerides. There are two strong absorption peaks at $2918 \mathrm{~cm}^{-1}$ and $2852 \mathrm{~cm}^{-1}$ that are characteristic of the asymmetric and symmetric stretching vibrations of methylene and also to methyl groups. Also, it is possible to observe two very defined peaks that could be related to the stretching vibration of $\mathrm{C}=\mathrm{O}$ and $\mathrm{CO}-$ of the ester group $\left(1742 \mathrm{~cm}^{-1}\right.$ and $1163 \mathrm{~cm}^{-1}$, respectively). The peaks at $1460 \mathrm{~cm}^{-1}$ and
$721 \mathrm{~cm}^{-1}$, represents the deformation vibration of $-\mathrm{CH}_{2}$ or $\mathrm{C}-\mathrm{H}_{3}$ groups, respectively, besides the rocking vibration in $\left(\mathrm{C}-\mathrm{H}_{2}-\right)_{\mathrm{n}}(\mathrm{n} \geq 4)$ groups. Moreover, the absorption bands at $3261 \mathrm{~cm}^{-1}$ and $3308 \mathrm{~cm}^{-1}$ could be associated with the stretching vibration of the hydroxyl group (O-H) (Han \& Wang, 2016; Ke et al., 2013).

Regarding rheological properties, organogels produced with HOSO and GM showed gel-like behavior with no frequency dependence and a prevalence of the elastic modulus through the whole frequency range (Fig. 2A and B). Moreover, the complex modulus increased with organogelator concentration increase, while $\tan \delta$ remained similar for all concentrations. These results are in agreement with other works that studied similar systems (Cerqueira et al., 2017). However, when such gels were subjected to shear at $1000 \mathrm{~min}^{-1}$ during $10 \mathrm{~min}$ in order to evaluate the effect of this step on their structure and their recovery ability after $24 \mathrm{~h}$, results showed that organogels were not able to fully recover their original structure. In fact, after mixing and $24 \mathrm{~h}$ recovery, organogels with $15 \%(\mathrm{w} / \mathrm{w}) \mathrm{GM}$ showed $G^{*}$ with the same magnitude of the original $5 \%(\mathrm{w} / \mathrm{w})$ system. This behavior was not expected since for low molecular weight organogelators (LMOGs) as GM, the driving forces involved are non-covalent, which confers a transient and dynamic character to the bonds thus forming physical gels (Patel, 2017; Sai Sateesh Sagiri, Singh, Kulanthaivel et al., 2015). Such result could be related to lower molecular mobility due to the higher viscosity at $25^{\circ} \mathrm{C}$, which could prevent the complete network recovery. In addition, all samples had similar $\tan \delta$ values, however, higher than the original systems (Fig. 2B).

On the other hand, the gelation mechanism of GG is a two-step process during cooling, where the random coil helices change to a double-helical conformation. After this coil-helix transition, the gellan double helix can aggregate to form junction zones. Hydrogen bonds between the junction zones can be induced at low $\mathrm{pH}$ values or with salt addition, resulting in macroscopic gel formation (Yamamoto \& Cunha, 2007). XRD results (Fig. 1A) confirmed that GG hydrogels evidenced an amorphous nature as previously reported (Arun Krishna \& Vishalakshi, 2017). GG FTIR spectra (Fig. 1B) showed absorption peaks at $1625 \mathrm{~cm}^{-1}$ and $1410 \mathrm{~cm}^{-1}$ due to asymmetric and symmetric stretching of the carboxylate group, while glycosidic bonds could be observed in the peaks between $1175-1140 \mathrm{~cm}^{-1}$. The band appearing at $1015 \mathrm{~cm}^{-1}$ is due to ethereal and hydroxylic $\mathrm{CO}-$ stretching. Moreover, in the same way as the organogels, GG present methylene and hydroxyl groups of glucopyranose ring. Thus, absorption peaks corresponding to these groups could be observed at $2888 \mathrm{~cm}^{-1}$ and in the $3259-3336 \mathrm{~cm}^{-1}$ band, respectively (Lee, Tsai, Wen, \& Huang, 2012; Sudhamani, Prasad, \& Udaya Sankar, 2003). However, the mentioned peaks were described for dry systems and care must be taken when analyzing hydrated hydrogels. The hydrogel could be microscopically named as a "two phases" system composed by water and the fibers network. The presence of water will interfere in the FTIR spectra (Ionita, 2016). Also, the water organization is different close to the fibers and in the solvent pools,
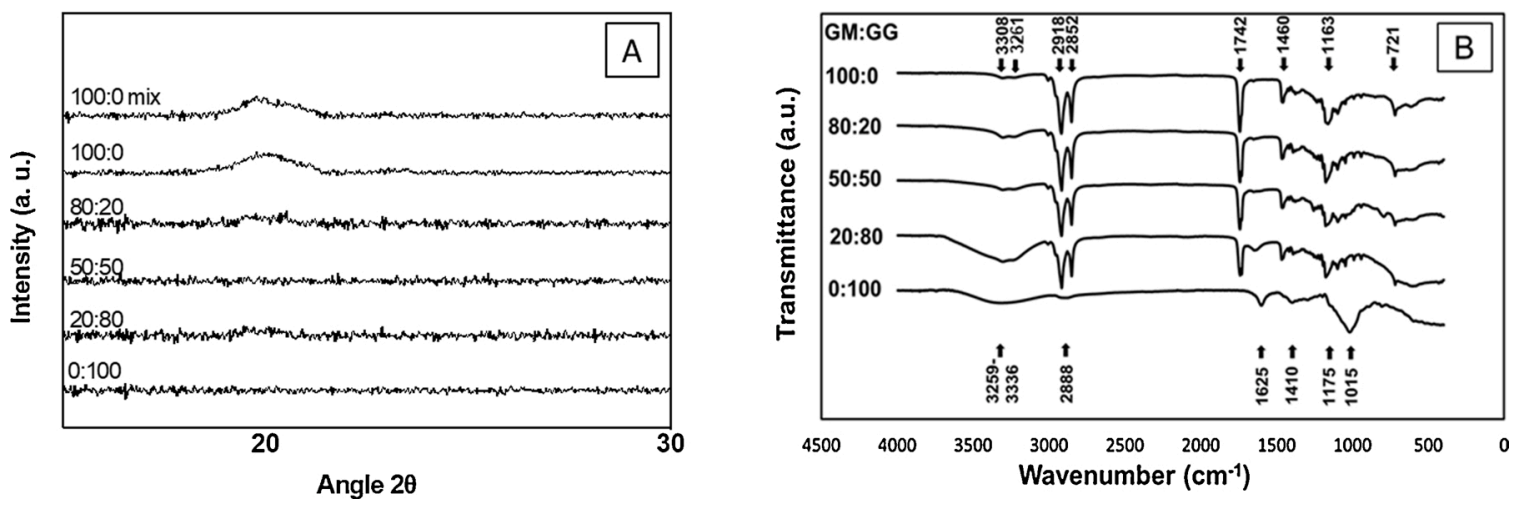

Fig. 1. XRD (A) and FTIR (B) spectra for pure organogel and hydrogel systems (original structure and mixed at $1000 \mathrm{~min}^{-1}$ for $10 \mathrm{~min}$ ) and bigels. 

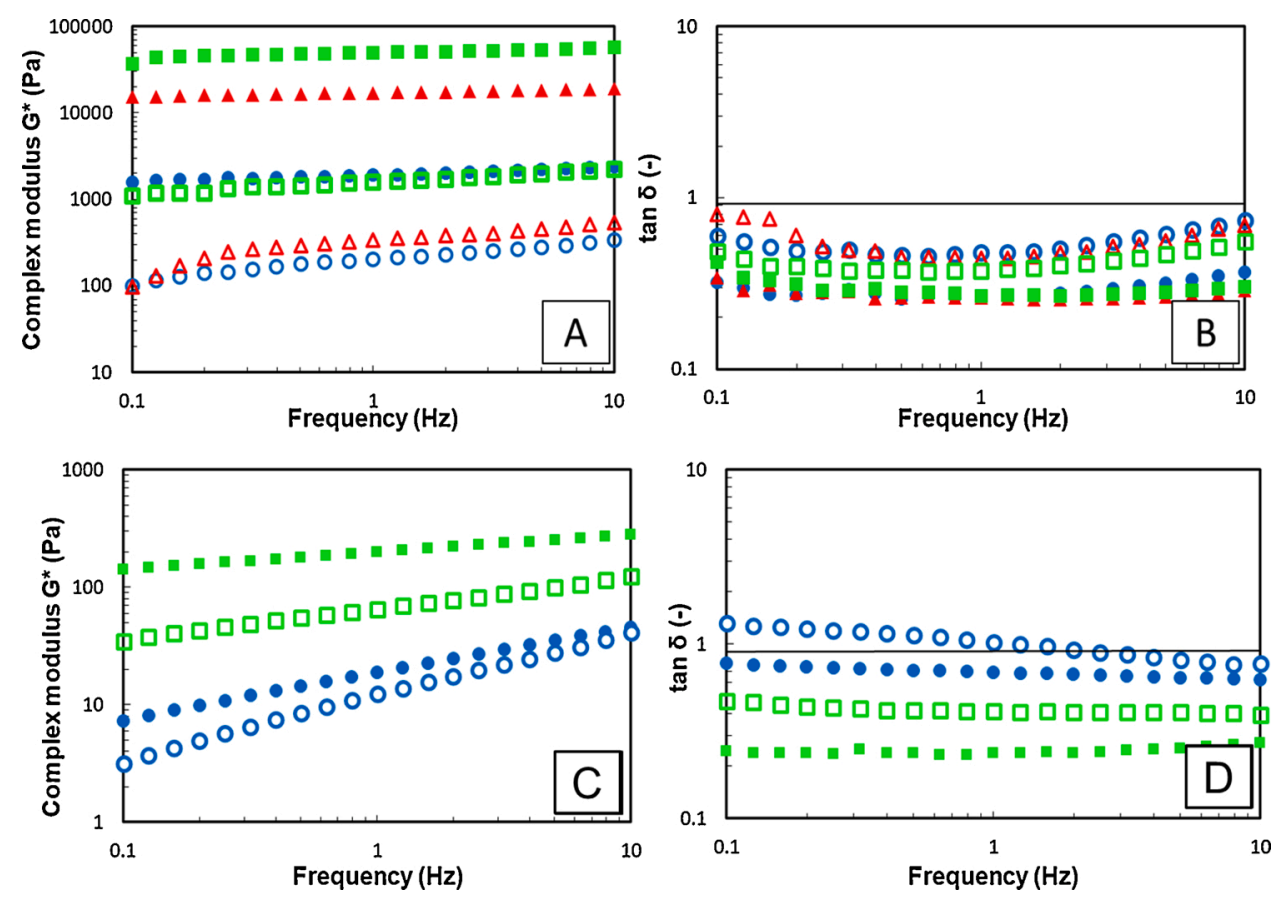

Fig. 2. Frequency sweep for pure organogels produced with HOSO and GM (A and B (O) $5 \%$ $(\mathrm{w} / \mathrm{w}) ;(\triangle) 10 \%(\mathrm{w} / \mathrm{w})$ and $(\square) 15 \%(\mathrm{w} / \mathrm{w}))$ and pure gellan gum hydrogel (C and D (O) $1 \%$ (w/w); ( ) $1.5 \%(w / w))$. (A) and (C) Complex modulus; (B) and (D) $\tan \delta$. Full symbols refer to the original organogels' structure after production and empty symbols to the recovered structure $24 \mathrm{~h}$ after mixing (shearing at 1000 $\min ^{-1}$ for $10 \mathrm{~min}$ ) (For interpretation of the references to colour in this figure legend, the reader is referred to the web version of this article).
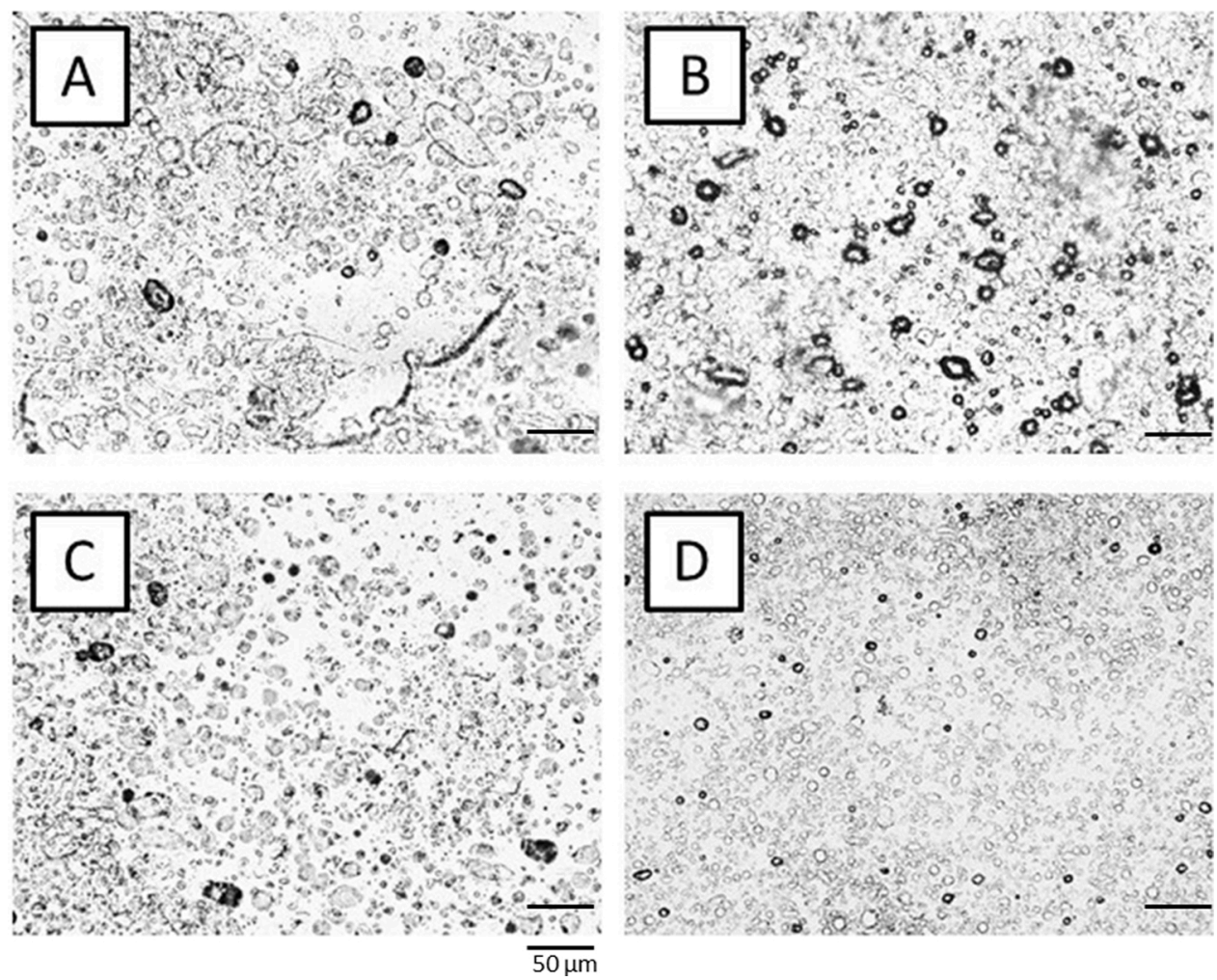

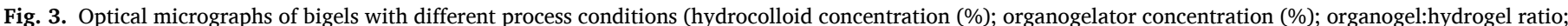

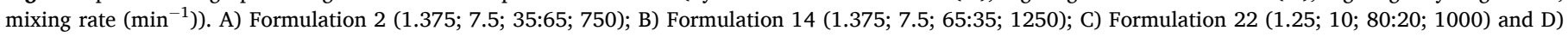
Formulation 27 (1.25; 10; 50:50; 1000). 
leading to the peaks that could be observed around $3200-3600 \mathrm{~cm}^{-1}$ band, including the free hydroxyl group (Cheng et al., 2013).

When mixed at the same conditions as the organogels $\left(1000 \mathrm{~min}^{-1}\right.$, $10 \mathrm{~min}$ ) the GG gels were broken into small particles. Fig. 2C and D show the behavior of broken GG gel against frequency for the lowest and highest gum concentrations studied. GG gels systems with $1 \%(w / w)$ of gellan gum lost their gel character and behaved as a concentrated solution showing frequency dependency (low values of $G^{*}$ and $\tan \delta$ close to unity). For a GG concentration of $1.5 \%(\mathrm{w} / \mathrm{w})$ the systems showed gel-like behavior, with $\tan \delta$ lower than 1 (prevalence of elastic modulus). However, this system showed lower $G^{*}$ values than the whole gel and a stronger frequency dependency (when compared to the whole gel). Increasing the mixing speed from 500 up to $1500 \mathrm{~min}^{-1}$ did not exert an effect on these results (data not shown).

All the results obtained for pure organogels and hydrogels are to be related later with bigel's properties. For example, since gellan broken gel (gellan particles) with $1.5 \%$ (w/w) of biopolymer are harder they could have more resistance to applied forces in comparison to the other gellan particles. On the other hand, FTIR and XRD could give insights about the phases' organization and interaction.

\subsection{General properties of bigels}

Bigels were evaluated in order to relate their microstructure to physical and textural properties. In order to represent and discuss the general behavior of bigels produced, patterns of XRD, FTIR, optical microscopy, rheological and mechanical analysis are represented in Figs. 1A, B, , respectively. Depending on the characteristics of original gels and the production method, bigels could be organized as oil-inwater, water-in-oil or bicontinuous systems. Fig. 3 shows that microstructure of bigels was composed by hydrogel particles dispersed in a continuous medium of organogel (water-in-oil structure). Other authors obtained W/O bigels when mixing the two previously gelled phases (Patel et al., 2015; Rehman et al., 2014; Rehman \& Zulfakar, 2017). However, O/W systems were also observed (Ibrahim, Hafez, \& Mahdy, 2013; Lupi et al., 2016), showing that the hydrogel and/or organogel network type influence the final structure. Other of the important factors is the temperature, since bigels produced by hot-emulsification processes led to O/W systems (Behera, Singh et al., 2015; Kodela et al., 2017). In this work, it could be unexpected the W/O structure even for high fractions of hydrogel. However, gellan gum formed a "hard" or very consistent gel at concentration above $1 \%(\mathrm{w} / \mathrm{w})$ that formed particles after shearing. On the other hand, organogels became a viscous liquid-like system, due to the nature of its weak physical arrangement. Therefore, we observe that the hidrogel broken into particles was surrounded by the "liquid" oleogel that had its structure rearranged with time. Also, in order to confirm such organization, we measured the electrical conductivity of the bigels that corroborates the visual observation with very low conductivity values (data not shown).

Fig. 1 shows XRD and FTIR spectra for some conditions used in hybrid systems preparation. Although the organogel maintains some crystallinity after shearing, bigels lost this feature with gellan ratio higher than $50 \%$, since the presence of gellan particles led to the prevalence of an amorphous character. The increase in GG concentration led to a lower crystallinity of the overall system. However, FTIR results showed that besides the crystallinity loss (observed in XRD analyses), bigels arrangement was formed only by physical interactions. Original peaks for pure GG and organogels were maintained and no new peaks or significant shifts were observed, which means that there were no new chemical bonds from the interaction between the gels. The complex physical arrangement (hydrogel particles dispersed in an organogel network) was dependent only on the composition and process conditions that exerted an effect on particles' size and consequently on the rheological and mechanical properties (Figs. 3 and 4).

Because of this complex microstructure, these biphasic gels present interesting rheological properties. Frequency sweeps (Fig. 4A) were similar to hybrid gels studied by other previous works (Lupi et al., 2016; Patel et al., 2015). All formulations showed gel-like behavior with a large range in the magnitude of the complex modulus $\left(G^{*}\right)$ and differences of up to three decades depending on the formulation (Fig. 4A). In addition, most systems did not show frequency dependence, however, the softer gels (lower $G^{*}$ values) presented some variations with increasing frequencies. Despite this, all bigels showed prevalence of elastic modulus ( $G^{\prime}$ ), i.e., $\tan \delta<1$ (Fig. 4B). Indeed, all $\tan \delta$ values were smaller than 0.4 at $1 \mathrm{~Hz}$.

It is important to highlight that the organic phase of bigels is composed of mixed organogels, i.e., which have a weaker structure in comparison to the original ones. However, in general, bigels were stronger than original organogels and hydrogels, with higher $G^{*}$ and lower $\tan \delta$ values, especially when the organogel content was higher than hydrogel. For example, pure $1.5 \%$ (w/w) GG and $15 \%$ (w/w) GM gels showed values of $G^{*}$ at $1 \mathrm{~Hz}$ of about $200 \mathrm{~Pa}$, and 50,000 $\mathrm{Pa}$, respectively (Table 2). On the other hand, most bigels had $G^{*}$ values higher than 50,000 Pa (Table 3). Such results suggest a synergistic effect between the two phases that enhances the rheological properties (i.e. led to stronger gels with higher $G^{*}$ and lower $\tan \delta$ ). Changes in network strength could be related to some interaction between the disperse and continuous phases. The hydrogel (dispersed phase) acted as filler in the structure, changing the physical properties of bigels. Such behavior has already been widely reported for emulgels describing the filler as active or inactive depending on its effect on the continuous phase (Dickinson, 2012). For bigels, due to the semi-solid state of both the internal and
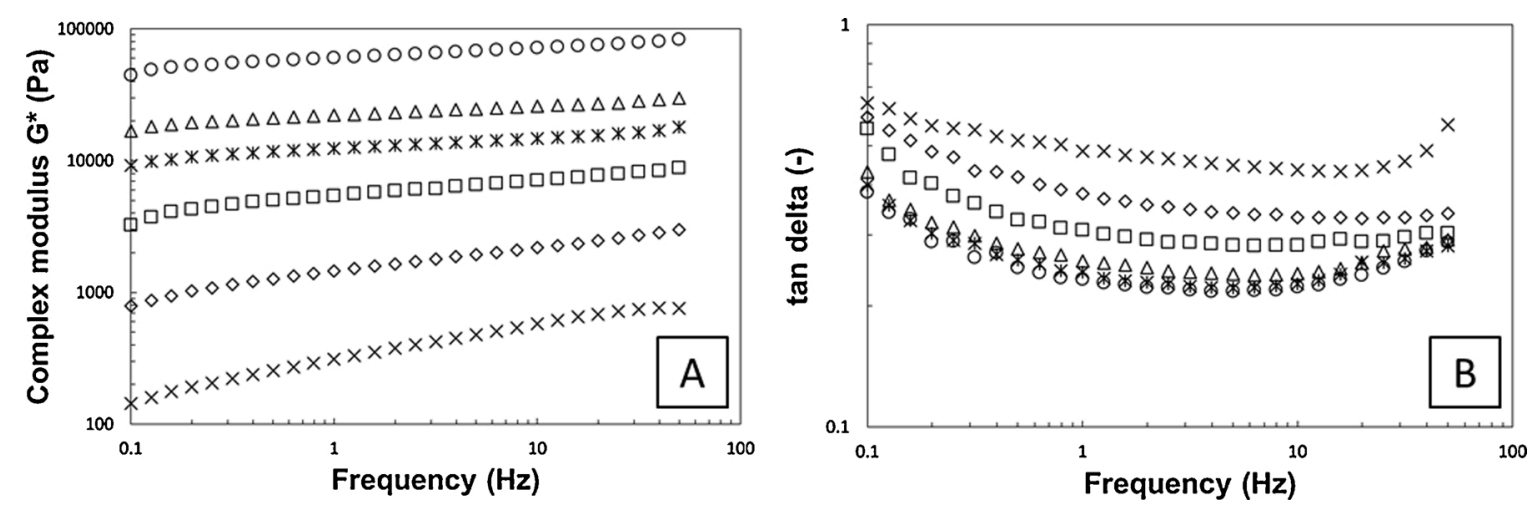

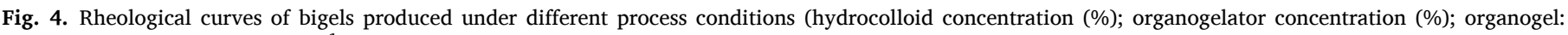

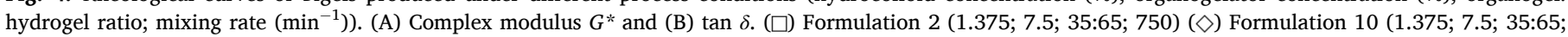

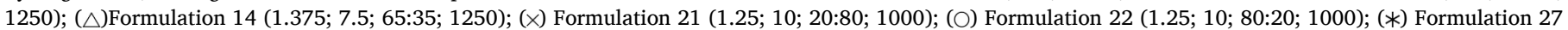
$(1.25 ; 10 ; 50: 50 ; 1000)$ (For interpretation of the references to colour in this figure legend, the reader is referred to the web version of this article). 
external phases, it is expected that its filler effect will be more pronounced (Wakhet et al., 2015). Patel et al. (2015) also observed a higher gel strength of a bigel compared to pure oleogel and hydrogel and the strengthening was more pronounced for higher fractions of oleogel. Similar behavior was described by Lupi et al. (2017) (2016), Singh, Anis et al. (2014) and Singh, Banerjee et al. (2014), where the disperse phase acted as an active filler, reinforcing the continuous phase strength. Zheng, Mao, Cui, Liu, and Gao (2020) also attributed the increase of the mechanical properties to the role of the oleogel network in the bigel. However, the opposite behavior was observed for sodium alginate hydrogel-beeswax oleogel bigels, where the increase of oleogel fraction disrupted the alginate network and consequently, decreased the mechanical properties (Martins et al., 2019).

Also, as mentioned before, organogels are self-organized and dynamic time-dependent systems, which means that the molecules' selfassembly demands some time to reach an equilibrium mesophase. Thus, this unsteady mesophase allows GM moieties mobility, especially after the bigel production. The GM moieties at the interface could selfassemble their glycerol hydrophilic head to the aqueous continuous phase, improving the affinity between the phases and reinforcing the network structure. In fact, for water-in-oil emulgels during the system formation, the emulsifier adsorbs as a film at the interface, enhancing the ability of the droplets to resist coalescence and as a consequence, act as an active filler (Rousseau, 2020).

Fig. 5 shows a representative textural profile of some bigels. The positive peak corresponds to hardness or firmness and its related to the resistance to deformation, i.e., its measured by the force required to obtain a given deformation. Work of adhesion or adhesiveness is the force needed to overcome the attractive forces between the sample and the probe contact area (herein we present negative values to indicate that the force is in a different direction, since the data was obtained in the post-test conditions when the probe moves upward). In addition, the work of shear was also calculated (in terms of total force required to carry out the shearing process). There is a significant interest in measuring the spreadability of food products such as butter, spreads, margarines and cheese, as well as non-food items such as lotions, creams, gel shaving creams, etc. Spreadability is a subjective property defined as the stress required to evenly distribute the product over a surface, i.e. the ability of the product to spread and deform with ease and uniformity (Daubert, Tkachuk, \& Truong, 1998). Although spreadability is also a deformation under an external load, it is a more dynamic property. Measurements of hardness and work of shear may be also indirect and inversely proportional to measurements of the spreadability of the samples, since a higher resistance to the applied forces (higher

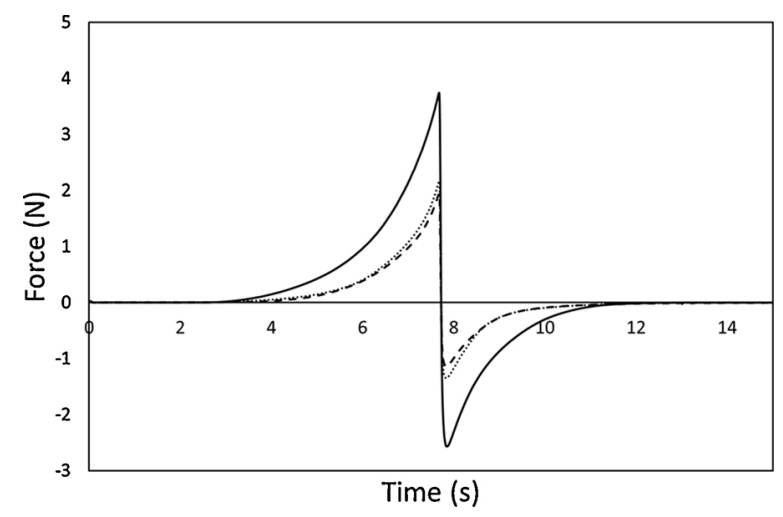

Fig. 5. Texture profile of bigels produced under different process conditions (hydrocolloid concentration (\%); organogelator concentration (\%); organogel: hydrogel ratio; mixing rate $\left(\min ^{-1}\right)$ ). (—) Formulation 7 (1.125; 12.5; 65:35; 750); (- - -) Formulation 1 (1.125; 7.5; 35:65; 750) and (. . .) Formulation 10 $(1.375 ; 7.5 ; 35: 65 ; 1250)$ (For interpretation of the references to colour in this figure legend, the reader is referred to the web version of this article). hardness and work of shear) makes the sample less spreadable.

Mechanical parameters (e.g. hardness, spreadability, adhesiveness) were dependent on the composition, as reported previously by other authors (Almeida et al., 2008; Behera, Dey et al., 2015; Rehman \& Zulfakar, 2017), and process parameter. In our work, these parameters were consistent with the rheological results for the gels produced. Systems with high $G^{*}$ also presented high hardness, work of shear and work of adhesion. In fact, bigels were able to withstand significantly higher stresses when compared to a one-component gel produced with the same amount (\% w/w) of gelator (GG or GM). For example, pure $1.5 \%(w / w)$ GG and $15 \%(w / w)$ GMmix gels showed hardness $(H d)$ values of about 1.2-1.3 N (Table 2), while bigels had $H d$ values higher than $2 \mathrm{~N}$, reaching even values higher than $10 \mathrm{~N}$ (Table 3). Once again, it is worth noticing the role of the particles acting as active filler, contributing to higher mechanical properties of the whole systems, as reported by other authors (Singh, Banerjee et al., 2014; Zheng et al., 2020).

\subsection{Statistical analysis (CCRD and PCA)}

Table 3 presents the results for the physical parameters of bigels according to the CCRD matrix. This table can give an idea of the effect exerted by an independent variable on the dependent variables. For example, increasing the hydrocolloid concentration generally leads to increasing values of hardness, adhesiveness, work of shear and $G^{*}$. Following the same preliminary analysis, increasing parameters like the organogelator concentration, the organogel:hydrogel ratio or the mixing rate, showed the same behavior for all the above-mentioned variables. Tan $\delta$ and diameter was also affected, but only by organogelator concentration and organogel:hydrogel ratio. In most cases, the diameter decreases with the increased of these variables, however, when the organogel fraction was higher but with lower organogelator concentration (i.e., higher fraction of a weak organogel), the diameter increased. Such tendency shows a synergistic effect between these two variables and reinforces the importance of the organogel network in the final structure. This synergism was corroborated by the statistical analyses in the following discussion.

Effects of the processing conditions on physical properties of bigels were evaluated and only considered relevant for a confidence level of 95 $\%(p<0.05)$. Significant effects were used to obtain empirical coded equations for adhesiveness, hardness, work of shear, $\tan \delta$, complex modulus and diameter (Table 3 ). In order to validate these empirical models at the assumed $5 \%$ significance level, an analysis of variance (ANOVA) was performed. This analysis showed that the coefficients of determination $\left(R^{2}\right)$ were greater than $90 \%$, except for $\tan \delta$ and diameter, for which values of near $80 \%$ were obtained (also good). Moreover, the values of $F_{\text {calc }}$ were higher than those tabulated, validating the empirical models. Thus, it was possible to generate the response surface for each response studied, as shown in Fig. 6.

Before evaluating response surfaces, it is important to note that not all parameters had a significant effect on the dependent variables studied. However, all parameters were a function of organogel:hydrogel ratio $(\mathrm{O}: \mathrm{H})$ and organogel concentration [Org]. For textural parameters (Adhesiveness $(A d)$, hardness $(H d)$, work of shear $(W s)$ ), both linear and quadratic effects and the interaction between these parameters were significant $(p<0.05)$, while for the other parameters only some were relevant. Mixing rate also exerted significant effect $(p<0.05)$ on the physical parameters, except for $\tan \delta$ and diameter, showing a quadratic effect for the mechanical parameters and some interaction with $\mathrm{O}: \mathrm{H}$ ratio in the complex modulus. On the other hand, hydrocolloid concentration had no effect on the variables within the limits studied $(p<$ 0.05).

Fig. 6 shows the response surfaces for the mean independent variables ([Org] and $(\mathrm{O}: \mathrm{H}))$ that exerted more significant effect according to Pareto's chart (data not shown) and were common for all dependent variables. Hardness and work of shear (Fig. 6A and B) showed very similar behavior with a curvilinear relationship between [Org] and $(\mathrm{O}$ : 

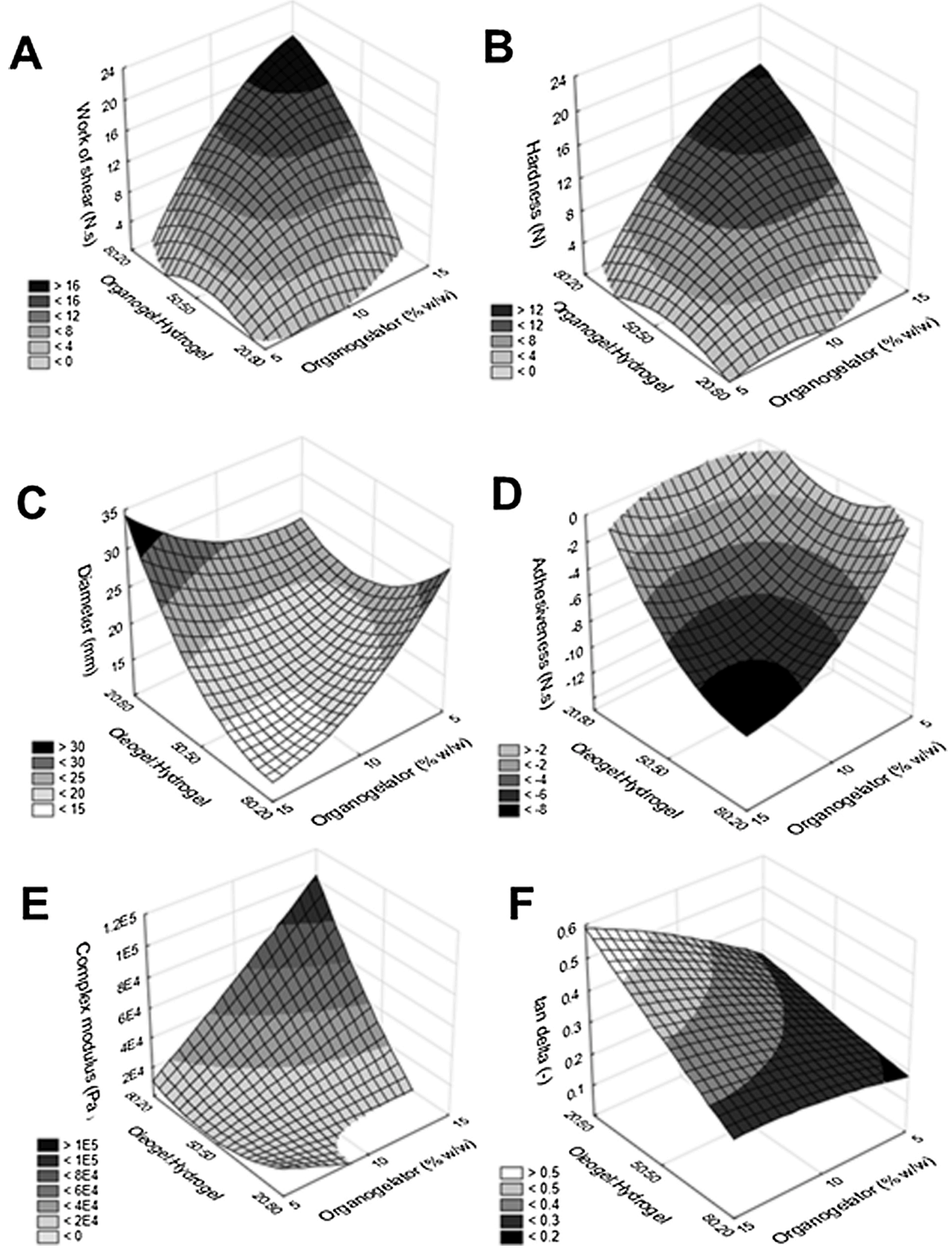

Fig. 6. Response surface for the physical parameters of bigels produced under different organogelator concentration and oleogel:hydrogel ratio. Mixing rate and hydrocolloid concentration was set at central point (1000 $\mathrm{min}^{-1}$ and $1.25(\% \mathrm{w} / \mathrm{w})$. (A) Work of shear; (B) Hardness; (C) Diameter; (D) Adhesiveness; (E) Complex modulus and $(\mathrm{F}) \tan \delta$. The $\mathrm{x}$ and $\mathrm{y}$ axes represent the parameters that exerted the greatest influence. 
H), reflecting the significant quadratic effect (Table 3). Increasing organogelator concentration led to higher values of these parameters; such behavior was more pronounced for higher organogel content $(\mathrm{O}: \mathrm{H}$ ratio).

A similar behavior was observed for the complex modulus (Fig. 6E), however, a significant increment occurred only for organogel concentrations above $50 \%$. The same was observed for adhesiveness (Fig. 6D): increasing organogelator concentration and organogel content led to more negative values, which means an increase of the adhesiveness. On the other hand, for the highest [Org] concentration and lowest organogel content or for the opposite combination (i.e. lowest [Org] and highest organogel content) the diameter increased (Fig. 6C). High friction and the consequent energy loss at the interface between the organogel and hydrogel phases could exert influence on the diameter reduction. However, these two extreme organogelator concentration and ratio combinations could lead to a decrease in the "global viscosity" of the organogel, decreasing the friction between the organogel and hydrogel phases during mixing and consequently producing particles with higher diameter. At last, $\tan \delta$ surface response (Fig. 6F) was quite linear, since there was only one significant quadratic effect exerted on this property. Higher $\tan \delta$ values or less predominance of the elastic character was observed with [Og] increase and organogel content decrease.

In general, it is possible to conclude that hydrogel particle size (physical arrangement) exerted an effect on the mechanical and rheological properties. Perhaps the key to understanding changes in rheological and mechanical properties is centered in this parameter. The effect of increasing organogel content and/or the occurrence of stronger structures (due to higher organogelator concentration) resulted in the ability to break the hydrogel in smaller particles, leading to a higher interface area. Thus, higher number of GM molecules could interact with the water particles at the interface, increasing the affinity between oil and water phases and reinforcing the structure of the bigel, as discussed before. Also, the dispersed particles droplet size and size distribution, as well as its fraction, will exert influence on the surface area between the particles and the continuous organogelled phase. Therefore, higher numbers of particles with smaller sizer distribution could act as an active filler, reinforcing substantially the structure. All these characteristics were reflected in an increase of hardness, work of shear, adhesiveness and complex modulus. In practice, response surface results show that it is possible to tailor their properties by understanding these effects and interactions and thus choosing the best levels of parameters.

Comparing with the results obtained in CCRD Pareto's diagram (data not shown), it is reliable to conclude that factor 1 (horizontal axis) represents the organogel:hydrogel ratio $(\mathrm{O}: \mathrm{H})$ and factor 2 (vertical axis) represents the organogelator concentration [Org]. The remaining $13 \%$ of the variability are represented only by mixing rate and the interactions between the parameters, since the CCDR showed that GG concentration did not exert a significant effect. From the projection of variables (see insertion of Fig. 7), it is possible to confirm that most of them were mainly responsible for the variability of data along the horizontal axis, except for $\tan \delta$, that is correlated to the vertical axis, i.e. related to organogelator concentration. Hardness and work of shear are well associated and also positively correlated to $\mathrm{G}^{*}$. On the other hand, diameter and adhesiveness are negatively correlated to these variables.

From the trials projected in Fig. 7 it was also possible to distinguish different groups with similar behavior. It is interesting, since a limited range in the values of the process parameters can lead to a product with specified characteristics. For example, Group E has an organogel content higher than $50 \%$ and organogelator concentrations between 10 and $12.5 \%(w / w)$. On the other hand, Group D was composed by trials with high organogel content ( $>50 \%$ ), but lower organogelator concentration $(<7.5 \% \mathrm{w} / \mathrm{w})$. However, this kind of analysis it not that simple, since there are interactions between the independent variables that must be further understood.

In any case, if we consider the application of bigels as replacers of fat content in foods, we can see that their physical properties are similar to those of some food products. Moreover, they can be used as the base material for cosmetic or pharmaceutical topical formulas and in all cases act as a vehicle for hydrophilic and lipophilic compounds. For example, some hard fat such as margarine or spreadable products show physical properties comparable to bigels of group A (Brighenti, Govindasamy-Lucey, Lim, Nelson, \& Lucey, 2008; Glibowski, Zarzycki, \& Krzepkowska, 2008; Yılmaz \& Öğütcü, 2014), that showed hardness higher than $3 \mathrm{~N}$ and $\mathrm{G}^{*}$ between 10,000 and 22,000 Pa. On the other hand, cosmetic formulations for skin care are similar to systems of group B (Calixto \& Maia Campos, 2017; Lukic, Jaksic, Krstonosic, Cekic, \& Savic, 2012), with hardness and $\mathrm{G}^{*}$ around $3 \mathrm{~N}$ and $2500 \mathrm{~Pa}$, respectively.

\section{Conclusions}

Bigels composed by a mixture of a hydrogel with an organogel were produced with gellan gum and high oleic sunflower oil with glycerol monostearate as organogelator. All hybrid systems were composed of hydrogel particles dispersed in an organogel continuous medium and

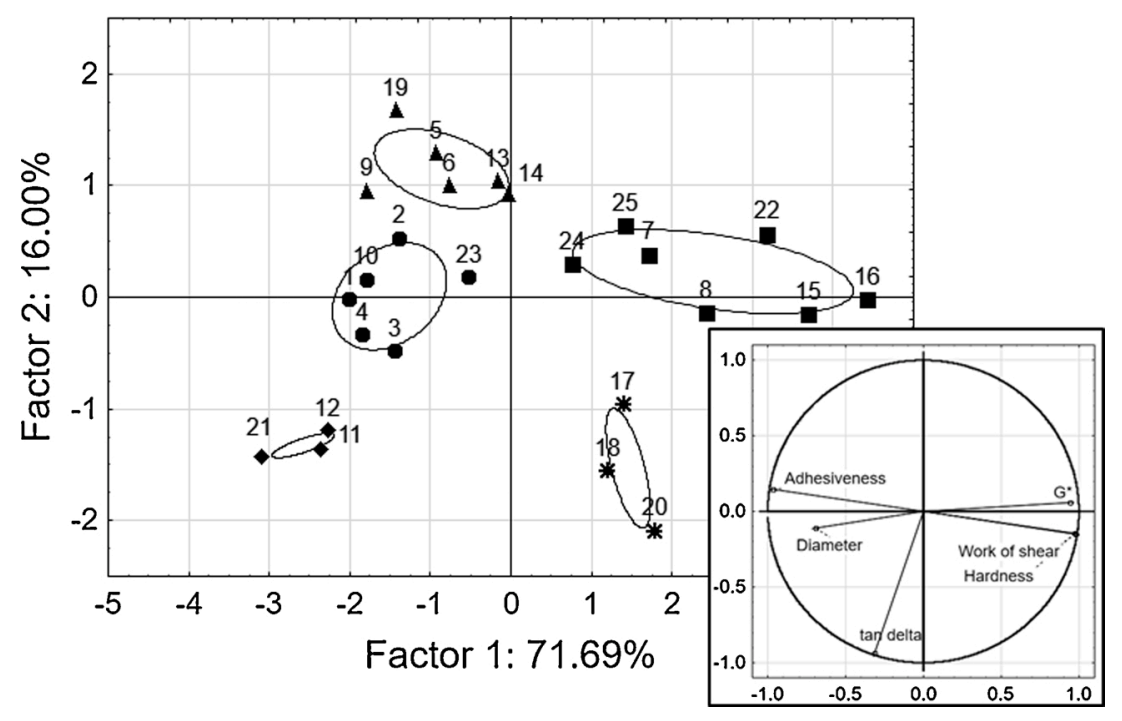

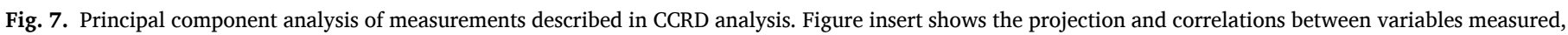
namely adhesiveness, diameter, tan delta, consistency, spreadability and complex modulus $\left(\mathrm{G}^{*}\right)$. 
showed gel-like behavior. However, all bigels showed a stronger network in comparison to pure organogel and hydrogel samples. This behavior could be mainly due to two phenomena: i) the dispersed hydrogel particles acted as an active filler ii) glycerol hydrophilic head of the organogelator presented at the interface self-assembled to interact with the water phase, improving the affinity between the oil-water phases. Moreover, bigels had harder or softer structures depending on their composition and processing conditions. Organogel:hydrogel ratio was the parameter that exerted the most significant influence on bigels' rheological, textural and structural properties, followed by organogelator concentration. The mixing rate and the interaction between the three parameters mentioned before were also significant. On the other hand, the hydrocolloid concentration was not a significant variable. In short, the decrease in the diameter of gellan particles led to higher interfacial area and consequently higher interaction of organogelator with the aqueous phase at the interface, improving the rheological and textural parameters: hardness, work of shear, adhesiveness and complex modulus. Bigels showed some characteristics that can be related to actual food, cosmetic and pharmaceutical products. Thus, bigels could be used to replace fat content in products like butter, cream cheese and other spreads, as well as to be used as the base material for body lotions and other topical products. This work showed that it is possible to tailor the texture and rheology of bigels, foreseeing their application in food products, by knowing how process variables are able to affect them.

\section{Acknowledgments}

This study was also supported by the Portuguese Foundation for Science and Technology (FCT) under the scope of the strategic funding of UID/BIO/04469/2013 unit and COMPETE 2020 (POCI-01-0145FEDER-006684) and BioTecNorte operation (NORTE-01-0145-FEDER000004) funded by the European Regional Development Fund under the scope of Norte2020 - Programa Operacional Regional do Norte. and of the Project RECI/BBB-EBI/0179/2012 (FCOMP-01-0124-FEDER027462).

\section{References}

Almeida, I. F., Fernandes, A. R., Fernandes, L., Pena Ferreira, M. R., Costa, P. C., \& Bahia, M. F. (2008). Moisturizing effect of oleogel/hydrogel mixtures. Pharmaceutical Development and Technology, 13(6), 487-494. https://doi.org/ 10.1080/10837450802282447.

Arun Krishna, K., \& Vishalakshi, B. (2017). Gellan gum-based novel composite hydrogel: Evaluation as adsorbent for cationic dyes. Journal of Applied Polymer Science, 134 (47), 45527. https://doi.org/10.1002/app.45527.

Behera, B., Sagiri, S. S., Singh, V. K., Pal, K., \& Anis, A. (2014). Mechanical properties and delivery of drug/probiotics from starch and non-starch based novel bigels: A comparative study. Standardization News, 66(9-10), 865-879. https://doi.org/ 10.1002/star.201400045.

Behera, B., Dey, S., Sharma, V., \& Pal, K. (2015). Rheological and viscoelastic properties of novel sunflower oil-span 40-biopolymer-based bigels and their role as a functional material in the delivery of antimicrobial agents. Advances in Polymer Technology, 34 (2), 21488. https://doi.org/10.1002/adv.21488.

Behera, B., Sagiri, S. S., Pal, K., Pramanik, K., Rana, U. A., Shakir, I., \& Anis, A. (2015) Sunflower oil and protein-based novel bigels as matrices for drug delivery applications-Characterization and in vitro antimicrobial efficiency. Polymer Plastics Technology and Engineering, 54(8), 837-850. https://doi.org/10.1080/ 03602559.2014 .974268$.

Behera, B., Singh, V. K., Kulanthaivel, S., Bhattacharya, M. K., Paramanik, K., Banerjee, I., \& Pal, K. (2015). Physical and mechanical properties of sunflower oil and synthetic polymers based bigels for the delivery of nitroimidazole antibiotic - A therapeutic approach for controlled drug delivery. European Polymer Journal, 64, 253-264. https://doi.org/10.1016/j.eurpolymj.2015.01.018.

Brighenti, M., Govindasamy-Lucey, S., Lim, K., Nelson, K., \& Lucey, J. A. (2008) Characterization of the rheological, textural, and sensory properties of samples of commercial US cream cheese with different fat contents. Journal of Dairy Science. https://doi.org/10.3168/jds.2008-1322.

Calixto, L. S., \& Maia Campos, P. M. B. G. (2017). Physical-mechanical characterization of cosmetic formulations and correlation between instrumental measurements and sensorial properties. International Journal of Cosmetic Science, 39(5), 527-534. https://doi.org/10.1111/ics.12406.

Carrillo-Navas, H., Pérez-Alonso, C., Fouconnier, B., Vernon-Carter, E. J., \& AlvarezRamírez, J. (2014). Inertial effects of adsorbed glycerol monostearate crystals on the shear rheology of water/canola oil interfaces. Journal of Food Engineering, 125, 112-118. https://doi.org/10.1016/j.jfoodeng.2013.10.025.

Cerqueira, M. A., Fasolin, L. H., Picone, C. S. F., Pastrana, L. M., Cunha, R. L., \& Vicente, A. A. (2017). Structural and mechanical properties of organogels: Role of oil and gelator molecular structure. Food Research International, 96. https://doi.org/ 10.1016/j.foodres.2017.03.021.

Cheng, F., Cao, Q., Guan, Y., Cheng, H., Wang, X., \& Miller, J. D. (2013). FTIR analysis of water structure and its influence on the flotation of arcanite (K2SO4) and epsomite (MgSO4. 7H2O). International Journal of Mineral Processing. https://doi.org/ 10.1016/j.minpro.2013.04.007.

Dassanayake, L. S. K., Kodali, D. R., Ueno, S., \& Sato, K. (2009). Physical properties of rice bran wax in bulk and organogels. Journal of the American Oil Chemists' Society, 86 (12), 1163-1173. https://doi.org/10.1007/s11746-009-1464-6.

Daubert, C. R., Tkachuk, J. A., \& Truong, V. D. (1998). Quantitative measurement of food spreadability using the vane method. Journal of Texture Studies. https://doi.org/ 10.1111/j.1745-4603.1998.tb00814.x.

Deng, G., Ma, Q., Yu, H., Zhang, Y., Yan, Z., Liu, F., ... Chen, Y. (2015). Macroscopic organohydrogel hybrid from rapid adhesion between dynamic covalent hydrogel and organogel. ACS Macro Letters, 4(4), 467-471. https://doi.org/10.1021/ acsmacrolett.5b00096.

Dickinson, E. (2012). Emulsion gels: The structuring of soft solids with protein-stabilized oil droplets. Food Hydrocolloids. https://doi.org/10.1016/j.foodhyd.2011.12.017.

Glibowski, P., Zarzycki, P., \& Krzepkowska, M. (2008). The rheological and instrumental textural properties of selected table fats. International Journal of Food Properties. https://doi.org/10.1080/10942910701622599.

Han, L., \& Wang, T. (2016). Preparation of glycerol monostearate from glycerol carbonate and stearic acid. RSC Advances, 6(41), 34137-34145. https://doi.org/ 10.1039/C6RA02912D.

Ibrahim, M. M., Hafez, S. A., \& Mahdy, M. M. (2013). Organogels, hydrogels and bigels as transdermal delivery systems for diltiazem HCL. Asian Journal of Pharmaceutical Sciences, 8(1), 46-54. https://doi.org/10.1016/j.ajps.2013.07.006.

Ionita, G. (2016). Characterization and tailoring the properties of hydrogels using spectroscopic methods. Emerging concepts in analysis and applications of hydrogels. https://doi.org/10.5772/62900.

Ke, H., Li, D., Wang, X., Wang, H., Cai, Y., Xu, Y., ... Wei, Q. (2013). Thermal and mechanical properties of nanofibers-based form-stable PCMs consisting of glycerol monostearate and polyethylene terephthalate. Journal of Thermal Analysis and Calorimetry, 114(1), 101-111. https://doi.org/10.1007/s10973-012-2856-7.

Kodela, S. P., Pandey, P. M., Nayak, S. K., Uvanesh, K., Anis, A., \& Pal, K. (2017). Novel agar-stearyl alcohol oleogel-based bigels as structured delivery vehicles. International Journal of Polymeric Materials and Polymeric Biomaterials, 66(13), 669-678. https://doi.org/10.1080/00914037.2016.1252362.

Lee, M.-W., Tsai, H.-F., Wen, S.-M., \& Huang, C.-H. (2012). Photocrosslinkable gellan gum film as an anti-adhesion barrier. Carbohydrate Polymers, 90(2), 1132-1138. https://doi.org/10.1016/j.carbpol.2012.06.064.

Lukic, M., Jaksic, I., Krstonosic, V., Cekic, N., \& Savic, S. (2012). A combined approach in characterization of an effective w/o hand cream: The influence of emollient on textural, sensorial and in vivo skin performance. International Journal of Cosmetic Science, 34(2), 140-149. https://doi.org/10.1111/j.1468-2494.2011.00693.x.

Lupi, F. R., Gentile, L., Gabriele, D., Mazzulla, S., Baldino, N., \& de Cindio, B. (2015). Olive oil and hyperthermal water bigels for cosmetic uses. Journal of Colloid and Interface Science, 459, 70-78. https://doi.org/10.1016/j.jcis.2015.08.013.

Lupi, F. R., Shakeel, A., Greco, V., Oliviero Rossi, C., Baldino, N., \& Gabriele, D. (2016). A rheological and microstructural characterisation of bigels for cosmetic and pharmaceutical uses. Materials Science and Engineering: C, 69, 358-365. https://doi. org/10.1016/j.msec.2016.06.098.

Lupi, F. R., De Santo, M. P., Ciuchi, F., Baldino, N., \& Gabriele, D. (2017). A rheological modelling and microscopic analysis of bigels. Rheologica Acta, 56(9), 753-763. https://doi.org/10.1007/s00397-017-1030-3.

Martins, A. J., Silva, P., Maciel, F., Pastrana, L. M., Cunha, R. L., Cerqueira, M. A., \& Vicente, A. A. (2019). Hybrid gels: Influence of oleogel/hydrogel ratio on rheological and textural properties. Food Research International, 116(July), 1298-1305. https:// doi.org/10.1016/j.foodres.2018.10.019.

Patel, A. R. (2017). A colloidal gel perspective for understanding oleogelation. Current Opinion in Food Science. https://doi.org/10.1016/j.cofs.2017.02.013.

Patel, A. R., Mankoč, B., Bin Sintang, M. D., Lesaffer, A., \& Dewettinck, K. (2015). Fumed silica-based organogels and "aqueous-organic" bigels. RSC Advances, 5(13), 9703-9708. https://doi.org/10.1039/C4RA15437A.

Rehman, K., Mohd Amin, M. C. I., \& Zulfakar, M. H. (2014). Development and physical characterization of polymer-fish oil bigel (hydrogel/oleogel) system as a transdermal drug delivery vehicle. Journal of Oleo Science, 63(10), 961-970. https://doi.org/ 10.5650/jos.ess14101.

Rehman, K., \& Zulfakar, M. H. (2014). Recent advances in gel technologies for topical and transdermal drug delivery. Drug Development and Industrial Pharmacy, 40(4), 433-440. https://doi.org/10.3109/03639045.2013.828219.

Rehman, K., \& Zulfakar, M. H. (2017). Novel fish oil-based bigel system for controlled drug delivery and its influence on immunomodulatory activity of imiquimod against skin cancer. Pharmaceutical Research, 34(1), 36-48. https://doi.org/10.1007/ s11095-016-2036-8.

Rousseau, D. (2020). Aqueous droplets as active fillers in oil-continuous emulsions. Current Opinion in Food Science. https://doi.org/10.1016/j.cofs.2020.06.004.

Sagiri, S. S., Singh, V. K., Kulanthaivel, S., Banerjee, I., Basak, P., Battachrya, M. K., \& Pal, K. (2015). Stearate organogel-gelatin hydrogel based bigels: Physicochemical, thermal, mechanical characterizations and in vitro drug delivery applications. Journal of the Mechanical Behavior of Biomedical Materials, 43, 1-17. https://doi.org/ 10.1016/j.jmbbm.2014.11.026. 
Sagiri, S. S., Singh, V. K., Pal, K., Banerjee, I., \& Basak, P. (2015). Stearic acid based oleogels: A study on the molecular, thermal and mechanical properties. Materials Science and Engineering C, 48, 688-699. https://doi.org/10.1016/j. msec.2014.12.018.

Sahoo, S., Singh, V. K., Uvanesh, K., Biswal, D., Anis, A., Rana, U. A., ... Pal, K. (2015) Development of ionic and non-ionic natural gum-based bigels: Prospects for drug delivery application. Journal of Applied Polymer Science, 132(38), 42561. https://doi. org/10.1002/app.42561.

Satapathy, S., Singh, V. K., Sagiri, S. S., Agarwal, T., Banerjee, I., Bhattacharya, M. K., .. Pal, K. (2015). Development and characterization of gelatin-based hydrogels, emulsion hydrogels, and bigels: A comparative study. Journal of Applied Polymer Science, 132(8). https://doi.org/10.1002/app.41502. n/a-n/a.

Shakeel, A., Farooq, U., Iqbal, T., Yasin, S., Lupi, F. R., \& Gabriele, D. (2019). Key characteristics and modelling of bigels systems: A review. Materials Science and Engineering: C, 97, 932-953. https://doi.org/10.1016/j.msec.2018.12.075.

Singh, V. K., Anis, A., Banerjee, I., Pramanik, K., Bhattacharya, M. K., \& Pal, K. (2014) Preparation and characterization of novel carbopol based bigels for topical delivery of metronidazole for the treatment of bacterial vaginosis. Materials Science and Engineering C, 44, 151-158. https://doi.org/10.1016/j.msec.2014.08.026.

Singh, V. K., Banerjee, I., Agarwal, T., Pramanik, K., Bhattacharya, M. K., \& Pal, K. (2014). Guar gum and sesame oil based novel bigels for controlled drug delivery. Colloids and Surfaces B: Biointerfaces, 123. https://doi.org/10.1016/j. colsurfb.2014.09.056.

Sudhamani, S., Prasad, M., \& Udaya Sankar, K. (2003). DSC and FTIR studies on Gellan and Polyvinyl alcohol (PVA) blend films. Food Hydrocolloids, 17(3), 245-250. https://doi.org/10.1016/S0268-005X(02)00057-7.
Toro-Vazquez, J. F., Morales-Rueda, J., Torres-Martínez, A., Charó-Alonso, M. A., Mallia, V. A., \& Weiss, R. G. (2013). Cooling rate effects on the microstructure, solid content, and rheological properties of organogels of amides derived from stearic and (R)-12- hydroxystearic acid in vegetable oil. Langmuir, 29(25), 7642-7654. https:// doi.org/10.1021/la400809a.

Trujillo-Ramírez, D., Lobato-Calleros, C., Jaime Vernon-Carter, E., \& Alvarez-Ramirez, J. (2019). Cooling rate, sorbitan and glyceryl monostearate gelators elicit different microstructural, viscoelastic and textural properties in chia seed oleogels. Food Research International, 119, 829-838. https://doi.org/10.1016/j. foodres.2018.10.066.

Wakhet, S., Singh, V. K., Sahoo, S., Sagiri, S. S., Kulanthaivel, S., Bhattacharya, M. K., ... Pal, K. (2015). Characterization of gelatin-agar based phase separated hydrogel, emulgel and bigel: A comparative study. Journal of Materials Science: Materials in Medicine, 26(2), 118. https://doi.org/10.1007/s10856-015-5434-2.

Yamamoto, F., \& Cunha, R. L. (2007). Acid gelation of gellan: Effect of final pH and heat treatment conditions. Carbohydrate Polymers, 68(3), 517-527. https://doi.org/ 10.1016/j.carbpol.2006.11.009.

Yılmaz, E., \& Ögüutcü, M. (2014). Comparative analysis of olive oil organogels containing beeswax and sunflower wax with breakfast margarine. Journal of Food Science, 79(9), E1732-E1738. https://doi.org/10.1111/1750-3841.12561.

Zheng, H., Mao, L., Cui, M., Liu, J., \& Gao, Y. (2020). Development of food-grade bigels based on $\kappa$-carrageenan hydrogel and monoglyceride oleogels as carriers for $\beta$-carotene: Roles of oleogel fraction. Food Hydrocolloids. https://doi.org/10.1016/j. foodhyd.2020.105855. 